J. Math. Kyoto Univ.

9-2 (1969) 161-188

\title{
On the Bott Suspension
}

\author{
By \\ I. M. JAMES \\ Dedicated to Professor A. Komatu on his sixtieth birthday
}

(Communicated by Professor H. Toda, November 5, 1968)

\section{Introduction}

In the original version [4] of the real periodicity theorem Bott introduces an operator into the structure of the homotopy groups of certain Lie groups. Following Harris [6] we refer to the operator as the Bott suspension. ${ }^{1)}$ In $\$ 1$ below we give a definition of the operator which is slightly different from Bott's and clarifies its relation with the Samelson product and other constructions. Our main purpose, however, is to introduce a relative version of the Bott suspension and to study its properties.

After some consideration of the general situation in which operators of this type arise we go on to make a detailed investigation of a particular example which operates on the homotopy groups of the Stiefel manifolds

$$
V_{2 n, 2 k}=S O(2 n) / S O(2 n-2 k) \quad(k \leqslant n) .
$$

In this case the relative Bott suspension constitutes a homomorphism

$$
F: \pi_{r}\left(V_{2 n, 2 k}\right) \rightarrow \pi_{r+1}\left(V_{2 n, 2 k}\right) .
$$

We fibre $V_{2 n, 2 k}$ over $V_{2 n, 2 l}(l \leqslant k)$ with fibre $V_{2 n-2 l, 2 k-2 l}$, in the usual way, and show that $F$ commutes ${ }^{2)}$ with the homomorphisms in the

1) The precise usage of Harris is somewhat different from mine. However, the name seems to be an appropriate one for any operator of the type.

2) As usual, in this kind of situation, there will be sign changes in some cases. 
homotopy exact sequence of the fibration.

There is a strong link between the Bott suspension and the homotopy theory of symmetric spaces. This enables us to calculate

$$
F: \pi_{r}\left(V_{2 n, 2}\right) \rightarrow \pi_{r+1}\left(V_{2 n, 2}\right),
$$

and hence obtain some information about $F$ in the general case. For example, we show that the iterated Bott suspension

$$
F^{q}: \pi_{r}\left(V_{2 n, 2 k}\right) \rightarrow \pi_{r+q}\left(V_{2 n, 2 k}\right)
$$

is trivial if $q \geqslant 6 k$; in particular

$$
F^{q}: \pi_{r}(S O(2 n)) \rightarrow \pi_{r+q}(S O(2 n))
$$

is trivial for $q \geqslant 6 n$. The actual results proved in $\S 5$ below are rather stronger than this.

The Bott suspension acts as a derivation with respect to the Samelson product, in a way we shall describe. Hence the calculations mentioned above enable us, in $\$ 7$, to compute a certain relative Samelson product which is the obstruction to the existence of a cross-section for a certain sphere-bundle (see [9] for details).

\section{The ordinary Bott suspension}

Let $G$ be a Lie group. Let $X$ be a 1-parameter subgroup of $G$ and let $X_{0} \subset X$ denote the cyclic subgroup given by integral values of the parameter. We describe $X$ as intercentral in $G$ if $X_{0}$ is contained in the centre of $G$. When this condition is satisfied the Bott suspension

$$
X_{\#}: \pi_{r}(G) \rightarrow \pi_{r+1}(G) \quad(r \geqslant 1)
$$

can be defined as follows, beginning with a special case.

Given an element $\theta \in \pi_{1}(G)$ we denote by

$$
\theta_{\sharp}: \pi_{r}(G) \rightarrow \pi_{r+1}(G)
$$

the homomorphism defined by taking the Samelson product with $\theta$, thus 


$$
\theta_{\sharp} \alpha=\langle\alpha, \theta\rangle \quad\left(\alpha \in \pi_{r}(G)\right) .
$$

When $X$ is such that $X_{0}$ is trivial we take $\theta$ to be the class of the loop determined by $X$ as the parameter runs from 0 to 1 and define $X_{\sharp}=\theta_{\xi}$.

In the general case, where $X$ is intercentral in $G$, we form the factor group $G^{\prime}=G / X_{0}$. The homomorphism $p_{*}: \pi_{r}(G) \rightarrow \pi_{r}\left(G^{\prime}\right)$ induced by the natural projection is an isomorphism for $r \geqslant 2$. Now $X_{0}^{\prime}$ is trivial, where $X^{\prime}=X / X_{0}$, and so $X_{\sharp}^{\prime}$ is defined as above. The Bott suspension associated with $X$ is defined by

$$
X_{\ddagger}=p_{*}^{-1} X_{\sharp}^{\prime} p_{*},
$$

as shown in the following diagram.

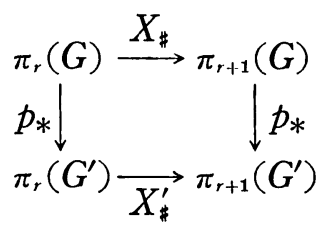

The usual definition of the Bott suspension involves a map $f: G \rightarrow \Omega G$, which we shall refer to as the Bott map associated with $X$. This map is defined by

$$
(f a)(t)=a x_{t} a^{-1} x_{t}^{-1} \quad(a \in G, t \in I),
$$

where $x_{t}$ denotes the element of $X$ given by parameter $t$. It is important to note that $f$ can be factored through $G / K$, where $K$ denotes the centralizer of $X$ in $G$. The Bott suspension is usually defined to be the composition

$$
\pi_{r}(G) \underset{f_{*}}{\longrightarrow} \pi_{r}(\Omega G) \underset{\xi}{\longrightarrow} \pi_{r+1}(G),
$$

where $\xi$ denotes the Hurewicz isomorphism. Then

$$
X_{\sharp}=\xi f_{*}
$$

by definition of the Samelson product when $X_{0}$ is trivial, and by naturality in the general case. 
Since $f$ is constant on $K$, the centralizer of $X$ in $G$, we obtain from (1.3) that

$$
X_{\sharp \sigma *}=0,
$$

where $\sigma_{*}: \pi_{r}(K) \rightarrow \pi_{r}(G)$ denotes the injection. To see this from our definition (1.1), consider the special case when $G=K$. Then $X_{\sharp}^{\prime}$ is trivial, since elements of the factor group $K / X_{0}$ commute with elements of $X^{\prime}$, and so $X_{\sharp}$ is trivial in this case. By naturality, therefore, $X_{\sharp} \sigma_{*}=\sigma_{*} X_{\sharp}=0$ in the general case, as asserted.

The standard example of the Bott suspension arises when $G=S O(2 n)$, the group of rotations of euclidean $2 n$-space. Take an orthonormal basis so that elements of $S O(2 n)$ are represented by matrices. We denote by

$$
F: \pi_{r}(S O(2 n)) \rightarrow \pi_{r+1}(S O(2 n))
$$

the Bott suspension associated with the 1-parameter subgroup $\exp (u \pi t)$, where $u$ denotes the matrix

$$
\left(\begin{array}{rr}
0 & 1 \\
-1 & 0
\end{array}\right) \oplus \cdots \oplus\left(\begin{array}{rr}
0 & 1 \\
-1 & 0
\end{array}\right) \quad(n \text { blocks }) .
$$

Note that the centralizer of the subgroup is the unitary group $U(n)$, embedded in the standard way. The factor group of $S O(2 n)$ by the central subgroup $\{e,-e\}$ is the projective group $P S O(2 n)$. The element $\theta \in \pi_{1}(\operatorname{PSO}(2 n))$ determined by $\exp (u \pi t)$ is of order 2 or 4 according as $n$ is even or odd. Hence it follows by linearity that

$$
\begin{cases}2 F=0 & (n \text { even }), \\ 4 F=0 & (n \text { odd }) .\end{cases}
$$

When $n$ is odd $2 \theta=p_{*} \varphi$, where $\varphi$ generates $\pi_{1}(S O(2 n))$, and so

$$
2 F=\varphi_{\sharp} \quad(n \text { odd }) .
$$

After this example we return to the general situation where $G$ is a Lie group and $X$ is an intercentral 1-parameter subgroup. I assert that $X_{\#}$ satisfies the derivation law

$$
X_{\#}\langle\alpha, \beta\rangle=\left\langle X_{\#} \alpha, \beta\right\rangle+(-1)^{i}\left\langle\alpha, X_{\sharp} \beta\right\rangle,
$$


where $\alpha \in \pi_{i}(G), \beta \in \pi_{j}(G)$. To see this, notice that

$$
p_{*}: \pi_{*}(G) \rightarrow \pi_{*}\left(G^{\prime}\right)
$$

respects the Samelson product, since $p$ is a homomorphism of topological groups. Now $X_{\sharp}^{\prime}$ acts as a derivation on $\pi_{*}\left(G^{\prime}\right)$, by the Jacobi identity for Samelson products. Since $p_{*}$ is an isomorphism of the higher homotopy groups it follows at once from (1.1) that $X_{\ddagger}$ acts as a derivation on $\pi_{*}(G)$, as asserted.

Next I assert that

$$
X_{\sharp} \circ Y_{\sharp}=-Y_{\ddagger} \circ X_{\sharp},
$$

where $X, Y$ are intercentral 1-parameter subgroups of $G$. Notice first that this relation holds when $X_{0}$ and $Y_{0}$ are trivial. For if $\theta, \varphi \in \pi_{1}(G)$ then $\langle\theta, \varphi\rangle \in \pi_{2}(G)=0$, and so

$$
\langle\langle\boldsymbol{\alpha}, \boldsymbol{\theta}\rangle, \varphi\rangle+\langle\langle\boldsymbol{\alpha}, \varphi\rangle, \theta\rangle=0,
$$

by the Jacobi identity, where $\alpha \in \pi_{*}(G)$. By taking $\theta, \varphi$ to be the elements determined by $X, Y$ we obtain (1.8) in the special case. To deal with the general case, consider the diagram of factor groups and natural projections shown below, where $G^{\prime \prime}$ denotes the factor group of $G$ by $X_{0} \cdot Y_{0}$.

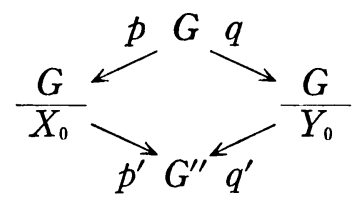

Now $X_{\#}$ is defined through $X_{\sharp}^{\prime}$, where $X^{\prime}=p X$, and $Y_{*}$ is defined through $Y_{\#}^{\prime}$, where $Y^{\prime}=q Y$. Write $X^{\prime \prime}=p^{\prime} X^{\prime}, Y^{\prime \prime}=q^{\prime} Y^{\prime}$. Then $X_{0}^{\prime \prime}$ and $Y_{0}^{\prime \prime}$ are trivial and so

$$
X_{\sharp}^{\prime \prime} \circ Y_{\sharp}^{\prime \prime}=-Y_{\sharp}^{\prime \prime} \circ X_{\sharp}^{\prime \prime},
$$

as shown above. We have $p_{*} X_{\sharp}=X_{*}^{\prime} p_{*}$, by definition of the Bott suspension, and $p_{*}^{\prime} X_{\sharp}^{\prime}=X_{\sharp}^{\prime \prime} p_{*}^{\prime}$, by naturality of the Samelson product. Hence $p_{*}^{\prime} p_{*} X_{\sharp}=p_{*}^{\prime} X_{\sharp}^{\prime} p_{*}=X_{\sharp}^{\prime \prime} p_{*}^{\prime} p_{*}$, and similarly $q_{*}^{\prime} q_{*} Y_{\sharp}=Y_{\sharp}^{\prime \prime} q_{*}^{\prime} q_{*}$. Now $p^{\prime} p=q^{\prime} q=r$, say, the natural projection of $G$ onto $G^{\prime \prime}$. The 
higher homotopy groups are mapped isomorphically by $r_{*}$. Hence the anticommutativity of the operators $X_{\#}, Y_{\#}$ follows from the anticommutativity of the operators $X_{\#}^{\prime \prime}, Y_{\sharp}^{\prime \prime}$.

When $X$ and $Y$ commute, we can construct the 1-parameter subgroup $Z \subset G$ such that $z_{t}=x_{t} \cdot y_{t}$, where $x_{t}, y_{t}, z_{t}$ are the elements of $X, Y, Z$ given by parameter $t$. I assert that

$$
X_{\sharp}+Y_{\sharp}=Z_{\sharp},
$$

when $X, Y, Z$ are so related. When $X_{0}$ and $Y_{0}$ are trivial this follows at once from the linearity of the Samelson product, since the element of $\pi_{1}(G)$ determined by $Z$ is the sum of the elements determined by $X$ and $Y$. To establish (1.9) in the general case we reduce it to the special case by an argument very similar to that used for (1.8). The details are omitted.

Of course (1.8) is almost obvious, when $X_{0}=Y_{0}$. For example, suppose that $X$ and $\lambda X$ commule, where $\lambda$ is an automorphism of $G$ which leaves $X_{0}$ fixed. Suppose that $X$ and $\lambda X$ commute. As $t$ runs from 0 to 1 we obtain from $X, \lambda X$ two paths from $e$ to a central element of $G$. By composing the first with the reverse of the second we obtain a loop in $G$, and it follows from (1.9) that

$$
X_{\#}-(\lambda X)_{\#}=\varphi_{\#},
$$

where $\varphi \in \pi_{1}(G)$ denotes the class of the loop. Now $\lambda$ determines an automorphism $\lambda^{\prime}$ of $G^{\prime}=G / X_{0}$, and we have a commutative diagram as shown below, from the definition of the Samelson product.

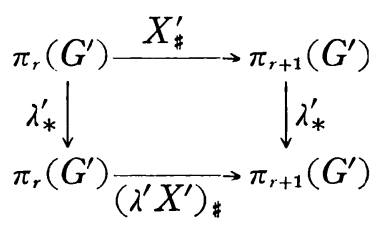

Hence it follows at once from (1.1) that $\lambda_{*} X_{\#}=(\lambda X)_{\#} \lambda_{*}$. Combining this with (1.10) we obtain the relation

$$
X_{\#}-\lambda_{*} X_{\#} \lambda_{*}^{-1}=\varphi_{\#} .
$$


As an example take $G=S O(4)$ with $\lambda$ the automorphism which changes the sign of the first row and column. This leaves $\{e,-e\}$ fixed and so (1.11) shows that

$$
F-\lambda_{*} F \lambda_{*}=\varphi_{*},
$$

where $\varphi$ generates $\pi_{1}(S O(4))$. We can use this relation to calculate

$$
F: \pi_{3}(S O(4)) \rightarrow \pi_{4}(S O(4)),
$$

as follows. Choose generators $\alpha^{\prime}, \beta^{\prime}$ of the cycle infinite groups $\pi_{3}(S O(3)), \pi_{3}(U(2))$ and let $\alpha, \beta \in \pi_{3}(S O(4))$ denote their images under the injections. Then $\lambda_{*} \beta=\alpha-\beta$, by (22.7) of [16], assuming that our choice of generators is as in [16]. Also $\psi_{\sharp} \beta^{\prime}=\beta^{\prime} \circ \eta$, by ${ }^{3)}$ Th. 1 of [5], where $\psi$ generates $\pi_{1}(U(2))$ and $\eta$ generates $\pi_{4}\left(S^{3}\right)$. Hence $\varphi_{*} \beta=\beta \circ \eta$, by naturality, and since $F \beta=0$, by (1.4), we obtain from (1.12) that

$$
-F \alpha=\lambda_{*}(\beta \circ \eta)=\alpha \circ \eta-\beta \circ \eta .
$$

This can also be established by the methods of $\$ 3$.

\section{The relative Bott suspension}

Before introducing the relative operator a further definition is required. Let $H$ be a closed subgroup of the Lie group $G$. We say that a 1-parameter subgroup $Y \subset H$ is subordinate to a 1-parameter subgroup $X \subset G$ if

$$
x_{t} h x_{t}^{-1}=y_{t} h y_{t}^{-1} \quad(h \in H),
$$

where $x_{t}, y_{t}$ denote the elements of $X, Y$ given by the parameter $t$. Let this condition be satisfied and let $X$ be intercentral in $G$. Then $Y$ is intercentral in $H$. Write $H \cdot X=\bar{H}$. Then $\bar{H}$ is a subgroup of $G$, and $H$ is a normal subgroup of $\bar{H}$. Also $\bar{H} / H$ is isomorphic to a factor group of $X$ and so its higher homotopy groups are trivial.

3) In the proof given in [5] there is a restriction imposed which appears to exclude this particular case. However, the restriction is not essential. 
Since $x_{t} y_{t}^{-1}$ lies in the centralizer of $H$, by (2.1), it follows from (1.4) and (1.9) that

$$
X_{\#}=Y_{\#}: \pi_{r}(\bar{H}) \rightarrow \pi_{r+1}(\bar{H}) .
$$

After making this observation we define the relative Bott suspension

$$
X_{\sharp}: \pi_{r}(G, H) \rightarrow \pi_{r+1}(G, H) \quad(r \geqslant 2)
$$

as follows. Write $\bar{H}^{\prime}=\bar{H} / X_{0}$, regarded as a subgroup of $G^{\prime}=G / X_{0}$. Then $X_{0}^{\prime}$ is trivial, where $X^{\prime}=X / X_{0}$ and so determines an element $\theta \in \pi_{1}\left(\bar{H}^{\prime}\right)$. Consider the homomorphism

$$
\theta_{\#}: \pi_{r}\left(G^{\prime}, \bar{H}^{\prime}\right) \rightarrow \pi_{r+1}\left(G^{\prime}, \bar{H}^{\prime}\right)
$$

defined by taking the relative Samelson product with $\theta$, thus

$$
\theta_{\sharp} \alpha=\langle\alpha, \theta\rangle \quad\left(\alpha \in \pi_{r}\left(G^{\prime}, \bar{H}^{\prime}\right)\right) .
$$

By the five lemma the induced homomorphism

$$
p_{*}: \pi_{r}(G, H) \rightarrow \pi_{r}\left(G^{\prime}, \bar{H}^{\prime}\right)
$$

is an isomorphism for $r \geqslant 2$. We define the relative Bott suspension $X_{\#}$ to be the homomorphism which makes the following diagram commutative.

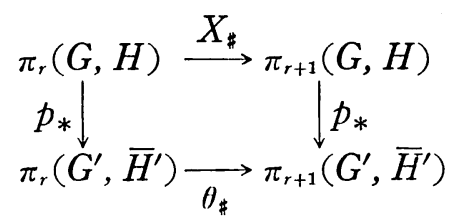

It is often convenient to identify the relative homotopy groups of the pair $(G, H)$ with the ordinary homotopy groups of the factor space $G / H$ under the isomorphism induced by the natural projection. Thus the (relative) Bott suspension can be regarded as a homomorphism of $\pi_{r}(G / H)$ into $\pi_{r+1}(G / H)$.

We recall from $\S 1$ of $[10]$ that $\theta_{\#}$ can be made to operate on every term of the homotopy exact sequence

$$
\cdots \rightarrow \pi_{r}\left(\overline{H^{\prime}}\right) \rightarrow \pi_{r}\left(G^{\prime}\right) \rightarrow \pi_{r}\left(G^{\prime}, \bar{H}^{\prime}\right) \rightarrow \cdots,
$$

raising dimension by 1 . On $\pi_{*}\left(\bar{H}^{\prime}\right)$ we take the Samelson product 
with $\theta$, on $\pi_{*}\left(G^{\prime}\right)$ we take the Samelson product with the image of $\theta$ in $\pi_{1}\left(G^{\prime}\right)$, and on $\pi_{*}\left(G^{\prime}, \bar{H}^{\prime}\right)$ we take the relative Samelson product, as above. By means of $p_{*}$ we transfer this to an operation of $X_{\#}$ on every term of the homotopy exact sequence

$$
\cdots \rightarrow \pi_{r}(H) \rightarrow \pi_{r}(G) \rightarrow \pi_{r}(G, H) \rightarrow \cdots .
$$

Here $\pi_{*}(G)$ is mapped by the ordinary Bott suspension and $\pi_{*}(G, H)$ by the relative. If we choose a subordinate subgroup $Y \subset H$ then the action of $X_{\#}$ on $\pi_{*}(H)$ agrees with that of the ordinary Bott suspension $Y_{\#}$. This follows at once from (2.2) since the injection

$$
\pi_{r}(H) \rightarrow \pi_{r}(\bar{H})
$$

is an isomorphism for $r \geqslant 2$.

Consider the Bott map $f: G \rightarrow \Omega G$ associated with $X$ as in (1.2). By (2.1) $f$ maps $H$ into $\Omega H$. Moreover $f \mid H$ agrees with the Bott map associated with $Y$, where $Y \subset H$ is subordinate to $X$. By a similar argument to the one used to prove (1.3) it can be shown that the following diagram is commutative, where $\xi$ denotes the relative Hurewicz isomorphism.

$$
\pi_{r}(G, \overbrace{X_{\sharp}^{H)}}^{f_{*}^{H}} \pi_{r}(\Omega G, \Omega H)
$$

Suppose, however, that we carry out the identification of $\pi_{*}(G, H)$ with $\pi_{*}(G / H)$. It might be conjectured that there exists a map of $G / H$ into $\Omega(G / H)$ such that $X_{\#}$ is the composite of the induced homomorphism and the Hurewicz isomorphism. In general this conjecture is false, as we shall see in $\$ 4$ below.

If $K$ is the centralizer of $X$ in $G$ then $H \cap K$ is the centralizer of $Y$ in $H$, where $Y$ is subordinate to $X$, and it follows as in the ordinary case that

$$
X_{\sharp} \sigma_{*}=0,
$$

as shown below, where $\sigma$ denotes the natural embedding of $K /(H \cap K)$ in $G / H$. 


$$
\pi_{r}(K /(H \cap K)) \underset{\sigma_{*}}{\longrightarrow} \pi_{r}(G / H) \underset{X_{\sharp}}{\longrightarrow} \pi_{r+1}(G / H)
$$

Now suppose that $H$ contains a closed subgroup $J$ of $G$ and that $J$ contains a 1-parameter subgroup subordinate to $Y$ and hence to $X$. Then the relative Bott suspension operates on the homotopy exact sequence of the triple $(G, H, J)$, mapping $\pi_{*}(G, H)$ and $\pi_{*}(G, J)$ according to $X_{\sharp}$ and $\pi_{*}(H, J)$ according to $Y_{\sharp}$. This follows at once from the characterization of the relative Bott suspension in terms of the Bott map.

An important example of the relative Bott suspension is when

$$
(G, H)=(S O(2 n), S O(2 n-2 k)),
$$

where $k \leqslant n$. Here we identify $S O(2 n-2 k)$ with the stability subgroup of the last $2 k$ basis vectors and identify the factor space $S O(2 n) / S O(2 n-2 k)$ with the (oriented) Stiefel manifold of orthonormal $2 k$-frames in $2 n$-space. We take $X$ to be the 1-parameter subgroup $\exp (u \pi t)$, as defined in $\S 1$, and $Y$ to be the corresponding 1-parameter subgroup of $S O(2 n-2 k)$, which satisfies the subordination condition (2.1). We denote by

$$
F: \pi_{r}\left(V_{2 n, 2 k}\right) \rightarrow \pi_{r+1}\left(V_{2 n, 2 k}\right)
$$

the Bott suspension thus defined. In this case $(K, H \cap K)=(U(n)$, $U(n-k))$ and the factor space $U(n) / U(n-k)$ can be identified with the complex Stiefel manifold $W_{n, k}$, regarded as a subspace of $V_{2 n, 2 k}$. By (2.3), therefore, $F$ annihilates the image of the injection

$$
\sigma_{*}: \pi_{r}\left(W_{n, k}\right) \rightarrow \pi_{r}\left(V_{2 n, 2 k}\right) .
$$

Consider the homotopy exact sequence of the fibration of $S O(2 n)$ over $V_{2 n, 2 k}$, with fibre $S O(2 n-2 k)$. We have defined $F$ on each term of the associated exact sequence so that $F$ constitutes a map of the exact sequence into itself. The same applies in the case of the fibration of $V_{2 n, 2 k}$ over $V_{2 n, 2 l}(l \leqslant k)$, with fibre $V_{2 n-2 l, 2 k-2 l}$.

Returning to the general situation we remark that the properties of the ordinary Bott suspension established in $\$ 1$ can be extended 
to the relative Bott suspension, with appropriate modifications, using similar arguments. For example, consider the derivation law (1.7), which depends on the Jacobi identity for the Samelson product. The Jacobi identity for the relative Samelson product, as proved in [14], gives rise to the derivation law

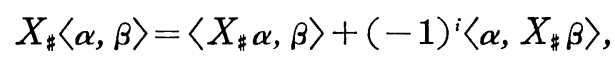

where $\alpha \in \pi_{i}(G, H), \beta \in \pi_{j}(H)$. If $Y \subset H$ is subordinate to $X \subset G$ then $X_{\sharp \beta} \beta$ can be changed to $Y_{\sharp \beta}$.

We recall from [13] that the Whitehead product in $\pi_{*}(G / H)$ can be expressed in terms of the relative Samelson product. Specifically, if $\alpha \in \pi_{i}(G / H), \gamma \in \pi_{j+1}(G / H)$ then $[\alpha, \gamma]=\langle\alpha, \Delta \gamma\rangle$, where

$$
\Delta: \pi_{j+1}(G / H) \rightarrow \pi_{j}(H)
$$

denotes the transgression operator in the homotopy sequence of the fibration. Since $X_{\#}$ commutes with $\Delta$ it follows from (2.4) that

$$
X_{\#}[\alpha, \gamma]=\left[X_{\sharp} \alpha, \gamma\right]+(-1)^{i}\left[\alpha, X_{\sharp \gamma}\right] .
$$

One further derivation law is worth mentioning, although it is not convenient to give the proof in the present paper. The intrinsic join operation, as defined in [7], constitutes a pairing of $\pi_{*}\left(V_{2 m, 2 k}\right)$ with $\pi_{*}\left(V_{2 n, 2 k}\right)$ to $\pi_{*}\left(V_{2 m+2 n, 2 k}\right)$. It can be shown that $F$ acts as a derivation with respect to this pairing.

\section{The Bott maps}

Let $G$ be a Lie group and let $X$ be an intercentral 1-parameter subgroup of $G$. Let $\lambda$ be an automorphism of $G$ such that the elements of $X$ commute with the elements of $Y=\lambda^{-1} X$. Then $Y$ forms an intercentral 1-parameter subgroup of $K$, the centralizer of $X$ in $G$. Let $L$ denote the centralizer of $Y$ in $K$. Consider the diagram shown below, where $\lambda^{\prime}$ is induced by $\lambda \mid K$ and $\mu$ is given by composition with $\lambda \mid K$. 


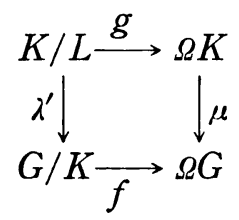

In the diagram $f$ is given by the Bott map associated with $X$, as defined in (1.2), and $g$ by the Bott map associated with $Y$. I assert that

$$
f \lambda^{\prime}=\mu g \text {. }
$$

For let $x_{t}$ denote the element of $X$ given by parameter $t$, and write $y_{t}=\lambda^{-1} x_{t}$. If $a \in K$ then

$$
\lambda\left(a y_{t} a^{-1} y_{t}^{-1}\right)=(\lambda a) x_{t}(\lambda a)^{-1} x_{t}^{-1},
$$

since $\lambda$ is an automorphism. Hence our assertion follows at once. It is important to notice that $\lambda^{\prime}$ is also induced by the map $\theta: K \rightarrow G$, where

$$
\theta a=(\lambda a) \cdot a^{-1} \quad(a \in K) .
$$

Let $M$ denote the subgroup of $K$ whose elements are left fixed by $\lambda$. Then

$$
\lambda^{\prime} q=p \theta^{\prime},
$$

as shown below, where $\theta^{\prime}$ is induced by $\theta$ and $p, q$ denote the natural projections.

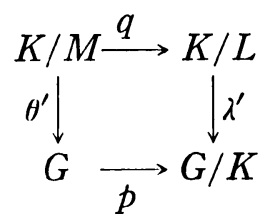

For the background to these constructions see Harris [6].

For example, take $G=S O(2 n)$, and take $X$ to be the 1-parameter subgroup $\exp (u \pi t)$ with centralizer $U(n)$. Choose $\lambda$ to be the involution which changes the sign of the first row and column. Then $Y$ is the 1-parameter subgroup $\exp (v \pi t)$, where $v$ is the matrix 


$$
\left(\begin{array}{rr}
0 & -1 \\
1 & 0
\end{array}\right) \oplus\left(\begin{array}{rr}
0 & 1 \\
-1 & 0
\end{array}\right) \oplus \cdots \oplus\left(\begin{array}{rr}
0 & 1 \\
-1 & 0
\end{array}\right)
$$

The centralizer of this subgroup of $U(n)$ is $U(1) \times U(n-1)$, and the factor space can be identified with $C P(n-1)$, the complex projective $(n-1)$-space. Moreover $M=O^{\prime}(1) \times U(n-1)$, where $O^{\prime}(1)$ denotes the subgroup of real matrices in $U(1)$, and so $K / M$ can be identified with $R P(2 n-1)$, the real projective $(2 n-1)$-space. Thus we have a commutative diagram as follows, where $W(n)$ $=S O(2 n) / U(n)$.

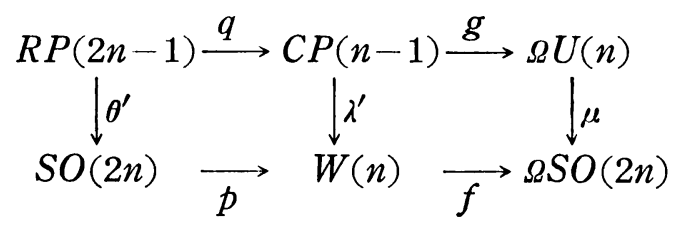

Here $\theta^{\prime}$ is the usual embedding (see [17]) of real projective space in the rotation group and $g$ is the adjoint (in the function-space sense) of the usual embedding (see [18]) of the suspension of complex projective space in the unitary group. The algebraic topology of $W(n)$ plays a central role in the theory of almost-complex structures. I remark in passing that the use of $\lambda^{\prime}$ considerably facilitates the study of this manifold.

It can easily be checked that the maps in the above diagram are compatible, for various values of $n$, if the usual embeddings are made. Thus the restriction of $\lambda^{\prime}$ to $C P(n-2)$ has values in $W(n-1)$ and agrees with the map obtained by our construction with $n-1$ in place of $n$. To avoid trivialities, take $n \geqslant 2$, from now on.

Consider the commutative diagram shown below, where the vertical maps are inclusions, $\theta^{\prime \prime}$ is determined by $\theta^{\prime}$, and $p^{\prime}$ denotes the natural projection. 


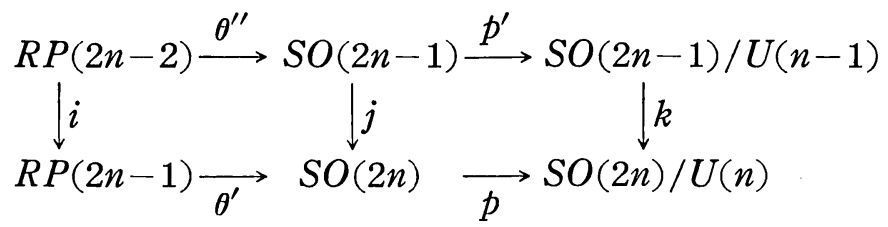

By (3.2) we have

$$
\lambda^{\prime} q i=p \theta^{\prime} i=p j \theta^{\prime \prime}=k p^{\prime} \theta^{\prime \prime} .
$$

Let $r^{\prime}: W(n) \rightarrow S^{2 n-2}$ denote the usual fibration, with fibre $W(n-1)$, as in $\S 41$ of $[16]$. We recall that $r^{\prime} k p^{\prime}=r$, the standard fibration of $S O(2 n-1)$ over $S^{2 n-2}$, and hence $r^{\prime} \lambda^{\prime} q i=r \theta^{\prime \prime}$, by (3.3). Now $r \theta^{\prime \prime}$ determines a relative homeomorphism

$$
(R P(2 n-2), R P(2 n-3)) \rightarrow\left(S^{2 n-2}, e\right),
$$

where $e$ denotes the basepoint, and $q$ determines a relative homeomorphism

$$
(R P(2 n-2), R P(2 n-3)) \rightarrow(C P(n-1), C P(n-2)) .
$$

Hence we obtain

Lemma 3. 4. The composition $r^{\prime} \lambda^{\prime}$ determines a relative homeomorphism

$$
(C P(n-1), C P(n-2)) \rightarrow\left(S^{2 n-2}, e\right) .
$$

In the present work the main purpose of (3.4) is to prove (3.5) below. Choose generators $\alpha_{n}^{\prime}, \beta_{n}^{\prime}$ of the infinite cyclic groups

$$
\pi_{2 n-2}(S O(2 n-1), S O(2 n-2)), \quad \pi_{2 n-1}(U(n), U(n-1)),
$$

where $n \geq 2$. Consider the elements

$$
\alpha_{n} \in \pi_{2 n-2}(S O(2 n), S O(2 n-2)), \quad \beta_{n} \in \pi_{2 n-1}(S O(2 n) . S O(2 n-2))
$$

which correspond to $\alpha_{n}^{\prime}, \beta_{n}^{\prime}$ under the injections. When these generators are suitably chosen $I$ assert that

$$
F \alpha_{n}=\lambda_{*} \beta_{n} \quad(n \geqslant 2),
$$

where $F$ denotes the relative Bott suspension and $\lambda_{*}$ the automorphism of $\pi_{*}(S O(2 n), S O(2 n-2))$ induced by $\lambda$. 
By (3.2) we have a commutative diagram as shown below, where we have identified $\pi_{r}(\Omega X, \Omega A)$ with $\pi_{r+1}(X, A)$ by the Hurewicz isomorphism.

$$
\begin{aligned}
\pi_{r}(C P(n-1), C P(n-2)) & \stackrel{\lambda_{*}^{\prime}}{\longrightarrow} \pi_{r}(W(n), W(n-1)) \\
g_{*} \downarrow & \stackrel{\downarrow}{\downarrow} f_{*} \\
\pi_{r+1}(U(n), U(n-1)) & \underset{\mu_{*}}{\longrightarrow} \pi_{r+1}(S O(2 n), S O(2 n-2))
\end{aligned}
$$

Take $r=2 n-2$. Then $g_{*}$ is an isomorphism, as shown in [18], and $\lambda_{*}^{\prime}$ is an isomorphism, by (3.4). Write $\gamma_{n}=g_{*}^{-1}\left(\beta_{n}^{\prime}\right)$. Then

$$
f_{*} \lambda_{*}^{\prime}\left(\gamma_{n}\right)=\mu_{*} g_{*}\left(\gamma_{n}\right)=\mu_{*}\left(\beta_{n}^{\prime}\right)=\lambda_{*}\left(\beta_{n}\right) .
$$

However $p_{*}\left(\boldsymbol{\alpha}_{n}\right)= \pm \lambda_{*}^{\prime}\left(\gamma_{n}\right)$, since

$$
p_{*}: \pi_{r}(S O(2 n), S O(2 n-2)) \rightarrow \pi_{r}(W(n), W(n-1))
$$

is an isomorphism when $r=2 n-2$. We choose $\alpha_{n}$ so as to make the sign positive and then obtain

$$
f_{*} \lambda_{*}^{\prime}\left(\gamma_{n}\right)=f_{*} p_{*}\left(\alpha_{n}\right)=F\left(\alpha_{n}\right),
$$

from the characterization of the Bott suspension in terms of the Bott map. Putting these relations together we obtain (3.5).

So far we have been working in terms of relative groups, but now we carry into effect our identification of $\pi_{r}(S O(2 n), S O(2 n-2))$ with $\pi_{r}\left(V_{2 n, 2}\right)$. We extend the meaning of $\lambda$ to include the induced automorphism of $V_{2 n, 2}$. It is shown ${ }^{4)}$ in $\S 6$ of [9] that

$$
\lambda_{*} \beta_{n}=\alpha_{n} \circ \eta-\beta_{n},
$$

where $\eta$ denotes the appropriate generator of the 1-stem. Therefore (3.5) implies that

$$
F \boldsymbol{\alpha}_{n}=\alpha_{n} \circ \eta-\beta_{n} .
$$

In this way we have determined the value of $F$ on the first nonvanishing homotopy group of $V_{2 n, 2}$. By naturality we can deduce

4) Note that $\lambda_{*}, \alpha_{n}, \beta_{n}$, in the present notation, correspond to $p_{*}, \alpha_{2 n}, \beta_{2 n}$, in the notation of [9]. 
the value of $F$ on the first non-vanishing homotopy group of $V_{2 n, 2 k}$ for $k \geqslant 1$. In all cases $\pi_{2 n-2 k}\left(V_{2 n, 2 k}\right)$ is infinite cyclic. When $k=1$ the image under $F$ has infinite order. When $k \geqslant 2$ the image under $F$ has order 2 or 4 according as $n-k$ is even or odd. This follows easily from (3.6) and elementary results on the homotopy groups of Stiefel manifolds (see [15], for example).

To conclude this section we consider the problem of whether there exists a map

$$
h: V_{2 n, 2} \rightarrow \Omega V_{2 n, 2}
$$

such that the following diagram is commutative, where $\xi$ denotes the Hurewicz isomorphism.

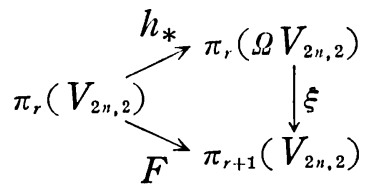

By (1.9) $F$ acts as a derivation on $\pi_{*}\left(V_{2 n, 2}\right)$, with respect to the Whitehead product. Since $F_{\beta_{n}}=0$, by (1.3), we obtain

$$
F\left[\alpha_{n}, \beta_{n}\right]=\left[F \alpha_{n}, \beta_{n}\right]=\left[\lambda_{*} \beta_{n}, \beta_{n}\right],
$$

by (3.5), and hence

$$
r_{*} F\left[\alpha_{n}, \beta_{n}\right]=\left[r_{*} \lambda_{*} \beta_{n}, r_{*} \beta_{n}\right],
$$

by naturality, where $r: V_{2 n, 2} \rightarrow S^{2 n-1}$ denotes the usual fibration. Now $r_{*} \beta_{n}$ and $r_{*} \lambda_{*} \beta_{n}$ are both generators of $\pi_{2 n-1}\left(S^{2 n-1}\right)$. Let $n \neq 2,4$. By Adams' theorem [1] the Whitehead product of these generators is non-zero, in $\pi_{*}\left(S^{2 n-1}\right)$, and so $F\left[\alpha_{n}, \beta_{n}\right]$ is non-zero, in $\pi_{*}\left(V_{2 n, 2}\right)$. But if $F=\xi h_{*}$, for some map $h$, then

$$
F\left[\alpha_{n}, \beta_{n}\right]=\xi\left[h_{*} \alpha_{n}, h_{*} \beta_{n}\right]=0,
$$

since $\pi_{*}\left(\Omega V_{2 n, 2}\right)$ has trivial Whitehead products, and so we have a contradiction.

Since $S O(2)=U(1) \subset U(2)$ there is a natural map of $V_{4,2}$ into $W(2)$. Hence it follows easily that $h$ exists when $n=2$. I do not know what the situation is when $n=4$. 


\section{The composition theorem}

It follows at once from our definition that the composition laws for the Bott suspension are the same as those for the corresponding Samelson product. Thus if $\mu \in \pi_{q}\left(S^{r}\right)$ then the ordinary Bott suspension satisfies the relation

$$
F(\theta \circ \mu)=F \theta \circ E_{\mu},
$$

where $E$ denotes the Freudenthal suspension. This relation also holds in the relative case, provided $\mu$ is a suspension element. These composition laws will be applied without further comment in what follows. Our main purpose in this section is to obtain an expression for the composition law in the relative case which is valid without restriction, and use this to compute

$$
F: \pi_{r}\left(V_{2 n, 2}\right) \rightarrow \pi_{r+1}\left(V_{2 n, 2}\right)
$$

in all dimensions. Not enough is known of the structure of $\pi_{*}\left(V_{2 n, 2 k}\right)$ with $k>1$ for any systematic result to be obtained but to some extent the same type of argument is applicable.

Let $\alpha_{n}, \beta_{n} \in \pi_{*}\left(V_{2 n, 2}\right)$ mean the same as in $\S 3$. Since $\beta_{n}$ is the class of a cross-section to the fibration of $V_{2 n, 2}$ over $S^{2 n-1}$ it follows at once that every element of $\pi_{r}\left(V_{2 n, 2}\right)$ can be expressed uniquely in the form

$$
\alpha_{n} \circ \varphi+\beta_{n} \circ \psi, \quad\left(\varphi \in \pi_{r}\left(S^{2 n-2}\right), \psi \in \pi_{r}\left(S^{2 n-1}\right)\right) .
$$

Since $F\left(\beta_{n} \circ \psi\right)=0$, by (1.4), the problem is to compute $F\left(\alpha_{n} \circ \varphi\right)$, using the expression for $F \alpha_{n}$ obtained in (3.6).

We begin with some observations on the Whitehead product structure of $\pi_{*}\left(V_{2 n, 2}\right)$, where $n \geqslant 2$. We have $\rho_{*} \alpha_{n}=0$, where

$$
\rho_{*}: \pi_{*}\left(V_{2 n, 2}\right) \rightarrow \pi_{*}\left(S^{2 n-1}\right)
$$

is induced by the fibration, and hence $\rho_{*}\left[\alpha_{n}, F \alpha_{n}\right]=0$ by naturality. Therefore

$$
\left[\boldsymbol{\alpha}_{n}, F \boldsymbol{\alpha}_{n}\right]=\boldsymbol{\alpha}_{n} \circ \mathrm{\xi},
$$


by exactness, for some (unique) element $\xi \in \pi_{4 n-4}\left(S^{2 n-2}\right)$. We shall prove that

$$
\left[\alpha_{n},\left[\alpha_{n}, F \alpha_{n}\right]\right]=0 .
$$

We recall from $\S 3$ of [10] that the composition law of Barcus and Barratt [3], originally proved for the Whitehead product, can be extended to the Samelson product as used in the definition of $F$. Thus we obtain an expression for $F\left(\alpha_{n} \circ \varphi\right)$ as a sum of terms beginning with $F_{\boldsymbol{\alpha}_{n}} \circ E_{\varphi}$. Each of the subsequent terms is the composite of a Whitehead product of the form

$$
\left[\alpha_{n}, F \alpha_{n}\right],\left[\alpha_{n},\left[\alpha_{n}, F \alpha_{n}\right]\right], \cdots,
$$

and some higher Hopf invariant of $\varphi$. Assuming (4.4) all these composite terms vanish, after the first, and so we obtain

Theorem 4.5. If $\varphi \in \pi_{r}\left(S^{2 n-2}\right)$ then

$$
F\left(\alpha_{n} \circ \varphi\right)=\left(F_{\alpha_{n}}\right) \circ\left(E_{\varphi}\right)+\alpha_{n} \circ \xi \circ E H \varphi,
$$

where $H_{\varphi} \in \pi_{r}\left(S^{4 n-5}\right)$ denotes the generalized Hopf invariant and $\xi$ is as in (4.3).

The proof of (4.4) depends on some information concerning $\xi$ which is also useful for the applications of (4.5) in $\$ 5$ below. In the homotopy sequence of the standard fibration of $U(n)$ over $S^{2 n-1}$, with fibre $U(n-1)$, the transgression carries a generator of $\pi_{2 n-1}\left(S^{2 n-1}\right)$ into an element $\mu^{\prime}$, say, of $\pi_{2 n-2}(U(n-1))$. We denote by $\mu \in \pi_{2 n-2}(S O(2 n-2))$ the image of $\mu^{\prime}$ under the injection. It is proved in (24.3) of [16] that $\rho_{*} \mu=\eta$ or 0 according as $n$ is odd or even, where now

$$
\rho_{*}: \pi_{2 n-2}(S O(2 n-2)) \rightarrow \pi_{2 n-2}\left(S^{2 n-3}\right)
$$

is induced by the standard fibration of $S O(2 n-2)$ over $S^{2 n-3}$. It is proved in (3.7) of $[11\rfloor$ that $\left[\alpha_{n}, \beta_{n}\right]=\alpha_{n} \circ J \mu$, where $J \mu$ denotes the element of $\pi_{4 n-4}\left(S^{2 n-2}\right)$ given by the Hopf construction. By (3.6), therefore 


$$
\begin{aligned}
{\left[\alpha_{n}, F \alpha_{n}\right] } & =\left[\alpha_{n}, \alpha_{n} \circ \eta\right]-\left[\alpha_{n}, \beta_{n}\right] \\
& =\alpha_{n} \circ\left(P_{\eta}-J \mu\right),
\end{aligned}
$$

where $P_{\eta}$ denotes the Whitehead product of $\eta \in \pi_{2 n-1}\left(S^{2 n-2}\right)$ with a generator of $\pi_{2 n-2}\left(S^{2 n-2}\right)$. Thus

$$
\xi=P_{\eta}-J \mu \text {. }
$$

We recall that $H P_{\eta}=0$ and $H J_{\mu}=E^{2 n-2} \rho_{*} \mu$. Hence it follows from (4.6) that

$$
\left\{\begin{aligned}
H \xi & =0 & & (n \text { even }), \\
& =\eta & & (n \text { odd }) .
\end{aligned}\right.
$$

By exactness $\mu^{\prime}$ lies in the kernel of the injection

$$
\pi_{*}(U(n-1)) \rightarrow \pi_{*}(U(n)),
$$

and so $\mu$ lies in the kernel of the injection

$$
\pi_{*}(S O(n-2)) \rightarrow \pi_{*}(S O(2 n) .
$$

Hence it follows that $E^{2} J \mu=0$ and so, since $E P_{\eta}=0$, we obtain the relation

$$
E^{2} \xi=0 \text {. }
$$

Hence, and from the composition law for the Whitehead product, we obtain that $P \xi=\gamma^{\circ} E^{2 n-2} H \xi$, where $\gamma \in \pi_{6 n-8}\left(S^{2 n-2}\right)$ denotes the triple Whitehead product of the generator of $\pi_{2_{n-2}}\left(S^{2 n-2}\right)$ with itself. But

$$
3 \gamma=0, \quad 2 H \xi=0,
$$

in all cases, and so $P \xi=0$. Since

$$
\left[\alpha_{n},\left[\alpha_{n}, F \alpha_{\alpha_{n}}\right]\right]=\left[\alpha_{n}, \alpha_{n} \circ \xi\right]=\alpha_{n} \circ P \xi
$$

this proves (4.4), and completes the proof of (4.5).

When $n=2$ we have $\xi=0$ and so (4.5) shows that (4.1) is valid without restriction in the case of $V_{4,2}$. This also follows from the existence of

$$
h: V_{4,2} \rightarrow \Omega V_{4,2},
$$

as described at the end of the previous section. 


\section{The iterated Bott suspension}

We begin by considering the operator

$$
F^{q}: \pi_{r}\left(V_{2 n, 2}\right) \rightarrow \pi_{r+q}\left(V_{2 n, 2}\right),
$$

for $q=1,2, \cdots$. It follows easily from (3.6) and the composition law that

$$
F^{4} \alpha_{n} \neq 0, \quad F^{5} \alpha_{n}=0,
$$

for $n \geqslant 2$. In the other direction we prove that

$$
\begin{cases}F^{5} \pi_{*}\left(V_{2 n, 2}\right)=0 & (n \text { even }), \\ F^{6} \pi_{*}\left(V_{2 n, 2}\right)=0 & (n \text { odd }) .\end{cases}
$$

I do not know of any example where $F^{5}$ is non-zero.

We deduce (5.2) from the composition theorem (4.5). Let $n$ be even. Then $H \xi=0$, by (4.7), and so $\xi$ is a suspension element. Hence and from (4.1) we obtain

$$
F^{2}\left(\alpha_{n} \circ \varphi\right)=F^{2} \alpha_{n} \circ E^{2} \varphi+F \alpha_{n} \circ E \xi \circ E^{2} H \varphi,
$$

by applying $F$ to both sides of the relation in (4.5). Now $E^{2} \xi=0$, by (4.8), and so when we apply $F$ again the second term on the right drops out and we are left with

$$
F^{3}\left(\alpha_{n} \circ \varphi\right)=F^{3} \alpha_{n} \circ E^{3} \varphi .
$$

Applying $F^{2}$ to both sides of this relation we obtain

$$
F^{5}\left(\alpha_{n} \circ \varphi\right)=F^{5} \alpha_{n} \circ E^{5} \varphi=0,
$$

by (5.1). This proves (5.2) when $n$ is even.

Now let $n$ be odd. From (4.5) with $\varphi=\xi$ we obtain

$$
F\left(\alpha_{n} \circ \xi\right)=F \alpha_{n} \circ E \xi+\alpha_{n} \circ \xi \circ E H \xi \text {. }
$$

Applying $F$ again we obtain

$$
F^{2}\left(\alpha_{n} \circ \xi\right)=F\left(\alpha_{n} \circ \xi\right) \circ E^{2} H \xi,
$$

since $E^{2} \xi=0$. However, $H \xi$ belongs to the 1 -stem and so by iteration we deduce from the above relation that $F^{5}\left(\alpha_{n} \circ \xi\right)=0$. Consequently 
when we apply $F^{5}$ to both sides of the relation in (4.5), with arbitrary $\varphi$, the second term drops out and we are left with

$$
F^{6}\left(\alpha_{n} \circ \varphi\right)=F^{6} \alpha_{n} \circ E^{6} \xi=0,
$$

by (5.1). This completes the proof of (5.2).

We return to the case when $n$ is even, and consider the injection

$$
i_{*}: \pi_{*}\left(V_{2 n, 2}\right) \rightarrow \pi_{*}\left(V_{2 n+2 k-2,2 k}\right),
$$

where $k \geqslant 2$. It is an elementary result (see [15]) in the homotopy theory of Stiefel manifolds that

$$
2 i_{*}\left(\beta_{n}\right)=\left(i_{*} \alpha_{n}\right) \circ \eta=i_{*}\left(\alpha_{n} \circ \eta\right) .
$$

Hence $i_{*}\left(\beta_{n}\right)=i_{*} F\left(\alpha_{n}\right)$, by (3.6). Since $F \beta_{n}=0$ this implies that

$$
i_{*} F^{2}\left(\alpha_{n}\right)=F i_{*} F\left(\alpha_{n}\right)=F i_{*}\left(\beta_{n}\right)=i_{*} F\left(\beta_{n}\right)=0 .
$$

Using this in (5.3) we obtain

$$
i_{*} F^{2}\left(\alpha_{n} \circ \varphi\right)=i_{*} F_{\alpha_{n}} \circ E \xi \circ E^{2} H \varphi .
$$

We apply $F$ once more and obtain

$$
i_{*} F^{3}\left(\alpha_{n} \circ \varphi\right)=i_{*} F^{2} \alpha_{n} \circ E^{2} \xi \circ E^{3} H_{\varphi}=0 .
$$

We change $n$ into $n-k+1$, for greater convenience at the next stage. Then the conclusion of the above argument is that

$$
i_{*} F^{3} \pi_{*}\left(V_{2 n-2 k+2,2}\right)=0 \quad(n-k \text { odd }),
$$

where $i_{*}$ now denotes the injection

$$
\pi_{*}\left(V_{2 n-2 k+2,2}\right) \rightarrow \pi_{*}\left(V_{2 n, 2 k}\right) .
$$

With this in hand we are now ready to prove

Theorem 5.5. Let $k \geqslant 2$. Then

$$
F^{q}: \pi_{r}\left(V_{2 n, 2 k}\right) \rightarrow \pi_{r+q}\left(V_{2 n, 2 k}\right)
$$

is trivial for $q \geqslant 9 k / 2+a_{n, k}$, where

$$
\begin{aligned}
a_{n, k} & =0 & & (n \text { odd }, k \text { even }), \\
& =1 / 2 & & (n \text { even, } k \text { odd }),
\end{aligned}
$$




$$
\begin{array}{ll}
=3 / 2 \quad & (n \text { odd, } k \text { odd }), \\
=2 & (n \text { even, } k \text { even }) .
\end{array}
$$

We proceed by induction on $k$, beginning with the case $k=2$. Consider the exact sequence

$$
\cdots \rightarrow \pi_{*}\left(V_{2 n-2,2}\right) \underset{i_{*}}{\longrightarrow} \pi_{*}\left(V_{2 n, 4}\right) \underset{j_{*}}{\longrightarrow} \pi_{*}\left(V_{2 n, 2}\right) \rightarrow \cdots
$$

associated with the standard fibration of $V_{2 n, 4}$ over $V_{2 n, 2}$ with fibre $V_{2 n-2,2}$. Let $\theta \in \pi_{*}\left(V_{2 n, 4}\right)$. Let $n$ be even, first of all. By naturality

$$
j_{*} F^{5} \theta=F^{5} j_{*} \theta=0,
$$

by (5.2), and so $F^{5} \theta=i_{*} \varphi$, by exactness, where $\varphi \in \pi_{*}\left(V_{2 n-2,2}\right)$. By naturality

$$
F^{6} i_{*} \varphi=i_{*} F^{6} \varphi=0,
$$

by (5.2), and so $F^{11} \theta=0$, as asserted. Now let $n$ be odd. In this case a similar argument shows that $F^{6} \theta=i_{*} \psi r$, where $\psi_{r} \in \pi_{*}\left(V_{2 n-2,2}\right)$. By naturality

$$
F^{3} i_{*} \psi=i_{*} F^{3} \psi=0,
$$

by (5.4), and so $F^{9} \theta=0$, as asserted. This completes the proof of (5.5) when $k=2$.

Now let $k>2$ and make the inductive hypothesis that (5.5) is true with $k-1$ in place of $k$. Consider the exact sequence

$$
\cdots \rightarrow \pi_{*}\left(V_{2 n-2 k+2,2}\right) \rightarrow \pi_{*}\left(V_{2 n, 2 k}\right) \rightarrow \pi_{*}\left(V_{2 n, 2 k-2}\right) \rightarrow \cdots
$$

associated with the standard fibration of $V_{2 n, 2 k}$ over $V_{2 n, 2 k-2}$ with fibre $V_{2 n-2 k+2,2}$. We argue in the same way as in the special case, using the inductive hypothesis on the right, and (5.2) or (5.4) on the left. The reader will easily check that the result is (5.5) as asserted. 


\section{Stable and non-stable results}

We begin with a result which is well-known, although there does not appear to be any suitable reference. Consider the ordinary Bott suspension

$$
F: \pi_{r}(S O(2 n)) \rightarrow \pi_{r+1}(S O(2 n)) .
$$

If $\theta$ belongs to the domain then

$$
F \theta=\theta \circ \eta \quad(r \leqslant 2 n-2),
$$

where $\eta$ generates the 1 -stem, as before. The basis for the proof is a result of Bott [4] that the kernel of $F$, in the stable range, coincides with the image of the injection

$$
\pi_{r}(U(n)) \rightarrow \pi_{r}(S O(2 n)) .
$$

Standard results on the stable homotopy groups of the classical groups show that this image coincides with the kernel of the homomorphism

$$
\eta^{*}: \pi_{r}(S O(2 n)) \rightarrow \pi_{r+1}(S O(2 n))
$$

given by composition with $\eta$. Consequently $F=\eta^{*}$, in the stable range. This proves (6.1) provided $r \leqslant 2 n-3$. When $r=2 n-2$ we consider the following naturality diagram, where $i_{*}$ denotes the injection.

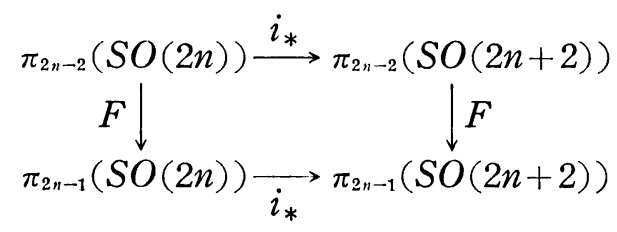

On the right we are within the stable range and so $F=\eta^{*}$, as we have seen. By naturality, therefore, $F \theta-\eta^{*} \theta$ lies in the kernel of $i_{*}$, on the bottom line of the diagram. However, the kernel is infinite cyclic while $\pi_{2 n-2}(S O(2 n))$ is a finite group. Consequently $F \theta=\eta^{*} \theta$, which completes the proof of (6.1). 
It is already clear from (1.13) that (6.1) breaks down outside the stable range. Let us examine the situation in the next two dimensions, using the results obtained by Kervaire [12].

Recall that $\alpha_{n+1}, \beta_{n+1}$, in $\S 3$, are derived from generators

$$
\alpha_{n+1}^{\prime} \in \pi_{2 n}(S O(2 n+2), S O(2 n)), \quad \beta_{n+1}^{\prime} \in \pi_{2 n+1}(U(n+1), U(n)) .
$$

Let $\lambda_{n} \in \pi_{2 n-1}(S O(2 n)), \mu_{n}^{\prime} \in \pi_{2 n}(U(n))$ denote the images of $\alpha_{n+1}^{\prime}, \beta_{n+1}^{\prime}$, respectively, under the boundary operator in the homotopy exact sequence of the pair. Then

$$
\Delta \alpha_{n+1}=\lambda_{n}, \quad \Delta \beta_{n+1}=\mu_{n},
$$

by naturality, where $\mu_{n}=\sigma_{*} \mu_{n}^{\prime} \in \pi_{2 n}(S O(2 n))$ and where

$$
\Delta: \pi_{r+1}\left(V_{2 n+2,2}\right) \rightarrow \pi_{r}(S O(2 n))
$$

denotes the boundary operator in the homotopy sequence associated with the standard fibration of $S O(2 n+2)$ over $V_{2 n+2,2}$ with fibre $S O(2 n)$. By (3.6) and naturality we have

$$
F \Delta \alpha_{n+1}=\Delta F \alpha_{n+1}=\Delta \alpha_{n+1} \circ \eta-\Delta \beta_{n+1},
$$

and so

$$
F \lambda_{n}=\lambda_{n} \circ \eta-\mu_{n}
$$

It is noteworthy that $\mu_{n}$, and hence $F \lambda_{n}$, has order 4 when $n$ is odd.

Similarly, by using the composition law, we can calculate the value of $F$ on any element in the image of $\Delta$. Since $F$ is trivial on the image of

$$
\sigma_{*}: \pi_{r}(U(n)) \rightarrow \pi_{r}(S O(2 n))
$$

by (1.4), it follows at once that $F$ can be calculated on the subgroup $\pi_{r}^{\prime}(S O(2 n))$ which is generated by the images of $\Delta$ and $\sigma_{*}$. When $n \neq 0 \bmod 4$ we find that

$$
\pi_{r}^{\prime}(S O(2 n))=\pi_{r}(S O(2 n))
$$

for $r=2 n-1,2 n$, and so these cases present no difficulty.

Let $n \equiv 0 \bmod 4$. Then the co-domain of 


$$
F: \pi_{r}(S O(2 n)) \rightarrow \pi_{r+1}(S O(2 n))
$$

is isomorphic to $Z_{2}+Z_{2}+Z_{2}$ for $r=2 n-1,2 n$. By combining the arguments indicated above with (6.1) it can be shown that the image of $F$ is of order 4 , for both these values of $r$. The details of the calculation are omitted.

\section{Sphere-bundles with $\boldsymbol{A}$-structure}

Let $X$ be a $(2 n+1)$-sphere bundle with group $S O(2 n+2)$. By an $A$-structure on $X$ we mean a fibre-preserving map $f: X \rightarrow X$ such that $f x$ is orthogonal to $x$ for all $x \in X$. By an almost-complex structure on $X$ we mean a reduction of the group of the bundle from $S O(2 n+2)$ to $U(n+1)$. Then the centre of $U(n+1)$ acts on $X$ and by defining $f x=u x$, where $u$ is central and $u^{2}=-e$, we obtain an $A$-structure from the almost-complex structure. As we have shown in [8], there exist bundles, even in the stable range, which admit an $A$-structure but not an almost-complex structure. The purpose of this section is to give an alternative proof of another result of [8] concerning the case when $X$ is stable and the base space is a sphere.

Theorem 7.1. Let $X$ be a $(2 n+1)$-sphere-bundle over $S^{r+1}$ with group $S O(2 n+2)$. Suppose that $r \leqslant 2 n-2$. Then $X$ admits an almost-complex structure if $X$ admits an A-structure.

We have shown in [9] how the condition for the existence of an $A$-structure can be expressed in terms of the Samelson product. In the following lemma we compute the relevant product, using the Bott suspension. Then we shall complete the proof of the theorem by appealing to the results of Adams [2] on the $J$-homomorphism.

The (relative) Samelson product determines a pairing of $\pi_{i}(S O(2 n))$ with $\pi_{j}\left(V_{2 n+2,2}\right)$ to $\pi_{i+j}\left(V_{2 n+2,2}\right)$. Consider the homomorphism

$$
\rho_{*}: \pi_{*}\left(V_{2 n+2,2}\right) \rightarrow \pi_{*}\left(S^{2 n+1}\right)
$$

induced by the usual fibration. We recall (see [13]) that 


$$
\rho_{*}\left\langle\theta, \beta_{n+1}\right\rangle=E J \theta,
$$

where $\theta \in \pi_{r}(S O(2 n))$ and where

$$
J: \pi_{r}(S O(2 n)) \rightarrow \pi_{r+2 n}\left(S^{2 n}\right)
$$

is given by the Hopf construction. Write

$$
\left\langle\theta, \beta_{n+1}\right\rangle=\alpha_{n+1} \circ \varphi+\beta_{n+1} \circ \psi
$$

as in (4.2), where $\varphi \in \pi_{r+2 n+1}\left(S^{2 n}\right), \psi \in \pi_{r+2 n+1}\left(S^{2 n+1}\right)$. It follows at once from (7.2) that $\psi=E J \theta$. I assert that

$$
\varphi=J(\theta \circ \eta) \quad(r \leqslant 2 n-2) .
$$

We have $F \theta=\theta \circ \eta$, by (6.1), and $F \beta_{n_{n+1}}=0$, by (1.4). Thus the derivation law yields

$$
F\left\langle\theta, \boldsymbol{\beta}_{n+1}\right\rangle=\left\langle\theta \circ \eta, \boldsymbol{\beta}_{n+1}\right\rangle=\left\langle\theta, \beta_{n+1}\right\rangle \circ \eta,
$$

by (4.1). Substituting from (7.3) at both ends of this relation we obtain

$$
F\left(\alpha_{n+1} \circ \varphi\right)=\alpha_{n+1} \circ \varphi \circ \eta+\beta_{n+1} \circ \psi \circ \eta \eta .
$$

By (3.6) and (4.1), however, we have

$$
F\left(\alpha_{n+1} \circ \varphi\right)=\alpha_{n+1} \circ \eta \circ E \varphi+\beta_{n+1} \circ E \varphi .
$$

Comparing coefficients of $\beta_{n+1}$ we obtain $E_{\varphi}=\psi \circ \circ \eta$, and hence $\varphi=J \theta \circ \eta$ $=J(\theta \circ \eta)$, as asserted, since we are in the stable range. This proves (7.4).

In (7.1) let $\theta^{\prime} \in \pi_{r}(S O(2 n+2))$ be the element corresponding to $X$ in the standard classification. Since $r \leqslant 2 n-2$ there exists a unique element $\theta \in \pi_{r}(S O(2 n))$ such that $\theta^{\prime}=i_{*} \theta$, where $i_{*}$ denotes the injection. Moreover $X$ admits an almost-complex structure if and only if $\theta$ lies in the image of $\sigma_{*}$, as shown in the following diagram.

$$
\pi_{r}(U(n)) \stackrel{\sigma_{*}}{\longrightarrow} \pi_{r}(S O(2 n)) \stackrel{\eta_{*}}{\longrightarrow} \pi_{r+1}(S O(2 n))
$$


By (4.4) and (5.1) of [9], the necessary and sufficient condition for the existence of an $A$-structure on $X$ is that

$$
\left\langle\theta, \beta_{n+1}\right\rangle=\beta_{n+1} \circ E J \theta .
$$

This relation holds, by (7.4), if and only if $J r^{*} \theta=0$. It is easy to show, from the periodicity tables and the results of Adams [2], that the image of $\sigma_{*}$ coincides with the kernel of $J \eta^{*}$ (as well as the kernel of $\eta^{*}$ ) in the stable range. Hence (7.1) follows at once.

It is not difficult to extend (7.1) to include the case $r=2 n-1$ but at present too little is known about the behaviour of Samelson products for us to make a substantial penetration into the nonstable range.

\section{References}

[1] J. F. Adams, on the non-existence of elements of Hopf invariant one, Ann. of Math. 72 (1960), 20-104.

[2] J. F. Adams, On the groups $J(X)$ IV, Topology 5 (1966), 21-71.

[3] W. D. Barcus and M. G. Barratt, On the homotopy classification of the extensions of a fixed map, Trans. Amer. Math. Soc. 88 (1958), 57-74.

[4] R. Bott, The stable homotopy of the classical groups, Ann. of Math. 70 (1959), 313-337.

[5] R. Bott. A note on the Samelson product in the classical groups, Comment. Math. Helv. 34 (1960), 249-256.

[6] B. Harris, Suspensions and characteristic maps for symmetric spaces, Ann. of Math. 76 (1962), 295-305.

[7] I. M. James, The intrinsic join, Proc. London Math. Soc. (3), 8 (1958), 507-535.

[8] I. M. James, Bundles with special structure I, Ann. of Math., 89 (1969), 359390.

[9] I. M. James, On fibre bundles and their homotopy groups. J. of Math. Kyoto Univ. 9 (1969), 5-24.

[10] I. M. James, Products between homotopy groups (to appear).

[11] I. M. James and J. H. C. Whitehead, The homotopy theory of sphere-bundles over sphere-bundles over spheres I. Proc. London Math. Soc. (3), 4 (1954), 196-218.

[12] M. A. Kervaire, Some non-stable homotopy groups of Lie groups, Illinois J. of Math. 4 (1960), 161-169.

[13] G. S. McCarty, Products between homotopy groups and the $J$-morphism, Quart. J. of Math. Oxford (2), 15 (1964), 362-370.

[14] G. S. McCarty, The value of $J$ at a Samelson product, Proc. Amer. Math. Soc. 19 (1968), 164-167.

[15] G. F. Paechter, The groups $\pi_{r}\left(V_{n, m}\right)$, Quart. J. of Math. Oxford (2), 7 (1956), 249-268. 
[16] N. E. Steenrod, The topology of fibre bundles, Princeton U. P. 1951.

[17] J. H. C. Whitehead, On the groups $\pi_{r}\left(V_{n}, m\right)$ and sphere-bundles, Proc. London Math. Soc. 48 (1944), 243-291.

[18] I. Yokota, On the cellular decompositions of unitary groups, J. of Inst. Poly. Osaka City Univ. 7 (1956), 39-49.

\section{OXFORD UNIVERSITY}

Mathematical Institute 